\title{
REMOVAL OF ABDOMINAL WALL FOR 3D VISUALIZATION AND SEGMENTATION OF ORGANS IN CT VOLUME
}

\author{
Feng DING, Wee Kheng LEOW* \\ Dept. of Computer Science \\ National University of Singapore \\ 13 Computing Drive, Singapore 117417
}

\author{
Sudhakar VENKATESH * \\ Dept. of Diagnostic Radiology \\ National University of Singapore \\ 10 Medical Drive, Singapore 117597
}

\begin{abstract}
$3 \mathrm{D}$ visualization and segmentation of organs in abdominal volume images are important in medical image processing for applications such as diagnosis, treatment and surgical planning. However, the abdominal wall leads to difficulties in both visualization and segmentation. These difficulties can be eliminated by removing the abdominal wall. This paper presents an algorithm that removes abdominal wall by registering a 3D flipping-free deformable model to the inner boundary of the wall. To our best knowledge, it is the first work in removing the abdominal wall for the purpose of 3D visualization and segmentation of the organs.
\end{abstract}

Index Terms - 3D deformable model, medical image segmentation, abdominal wall

\section{INTRODUCTION}

$3 \mathrm{D}$ visualization and segmentation of organs in abdominal volume images are important in medical image processing for applications such as diagnosis, treatment and surgical planning. However, the abdominal wall leads to difficulties in both visualization and segmentation. For visualization, the existence of the abdominal wall which consists of skin, fat, muscle and bones prevents the viewer from examining the organs in the volume rendered 3D image. A common solution in practice is to apply a transfer function for the user to adjust the opacity of the rendered volume. Although adjusting transparency of the abdominal wall voxels enables the user to see some of the organs, it can also cause organ voxels to appear transparent and cannot be clearly visible. The organs can physically touch the abdominal wall, and their voxel intensities are close to those of the abdominal wall. Such properties lead to unclear boundaries between organs and the abdominal wall, which leads to difficulty in 3D segmentation.

These problems can be eliminated by removing the abdominal wall. It usually contains only 4 types of tissues, i.e., skin, fat, muscle, and bones (ribs and spines), and they usually

*This research is supported by SBIC RP C-008/2006 and A*STAR SERC 0521010103 (NUS R-252-000-319-305). have different x-ray attenuation coefficients. So, the abdominal wall is easier to be modelled using its intensity distribution. This motivates our research on abdominal wall removal to facilitate $3 \mathrm{D}$ visualization and segmentation of the organs.

\section{RELATED WORK}

General deformable segmentation algorithms such as snake [1] and level set $[2,3]$ are not appropriate for extracting the abdominal wall. The snake algorithm, which deforms a contour based on strong edge information, may be attracted to noise edges instead of the true boundary of the wall. The level set algorithm adopts an implicit representation and is computationally expensive. It is also prone to the leakage problem. Moreover, both methods are difficult to be initialized for extracting the wall.

Maeda et al. [4] proposed to use rib cage to approximate the interface between the abdominal wall and the cavity. Spline interpolation is used to estimate the position of the interface where ribs are missing. A user interface is used to initialize a liver model where the edge between the liver and the abdominal wall is weak. This approach can work to some extent. However, it is in general not accurate enough to approximate the anterior part of the abdominal wall where the rib cage is made up of coastal cartilage. The cartilage has very similar x-ray attenuation coefficient to that of the neighboring muscles, and thus is difficult to be identified. Interpolation in these regions is inaccurate. Therefore, rib cage alone cannot provide enough information for extracting the abdominal wall. Moreover, their method requires that the rib cage be segmented first. Due to highly variant bone density across different patients and noise in the CT volume, simple algorithms such as thresholding are not robust and accurate enough for segmenting spine/ribs. In contrast, the proposed algorithm does not require accurate segmentation of the rib cage, though a relative good segmentation of these bones can help to extract the abdominal wall more accurately. Existing rib cage segmentation algorithms such as [5] can be applied.

The proposed algorithm requires the user to initialize a generic mesh sphere inside the body volume. This can be 


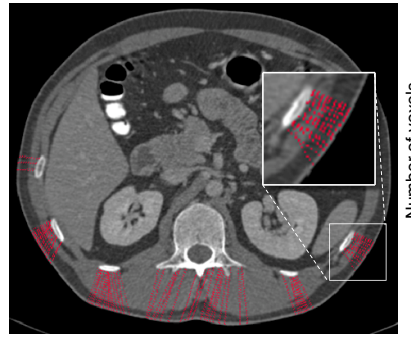

(a)

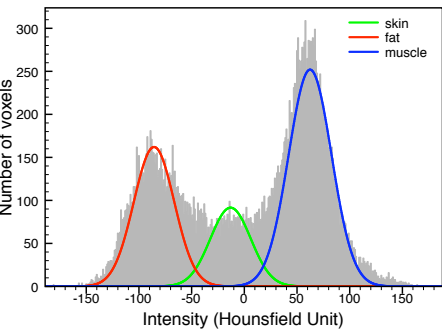

(b)
Fig. 1. (a) Extract and sample feature points (red) in the abdominal wall for (b) building intensity GMM.

easily automated by putting an appropriately sized spherical mesh at the center of gravity of the volume data. Based on the constructed intensity distribution of the abdominal wall (Section 3.2), the mesh model is deformed iteratively to register to the inner boundary of the abdominal wall (Section 3.3). To our best knowledge, this is the first work in removing the abdominal wall for the purpose of 3D visualization and segmentation of the organs.

\section{METHOD}

\subsection{Overview of Algorithm}

Our algorithm extracts the abdominal wall by i) image feature extraction from the input volume and ii) 3D flipping-free deformation of a generic mesh model to register it to inner boundary of the abdominal wall.

\subsection{Feature Extraction}

The feature extraction stage estimates the intensity distribution of abdominal wall voxels. Since voxels between the skin surface and the bone structure clearly belong to the abdominal wall, they can be sampled to build an intensity distribution to approximate that of the abdominal wall. Therefore, the feature extraction stage contains two steps. The first is to identify the skin surface and the bones, and the second is to build the intensity distributions of the body wall voxels.

Identification of skin surface and bones. Identification of the skin surface is straight-forward by using a contour tracing algorithm [6]. Since accurate segmentation of the bone structure is not necessary, identification of bone voxels can simply be performed by applying thresholding with a high threshold and then by extracting the largest connected component. Note that thresholding alone may get voxels belonging to other body parts as well besides bones. These parts have similar or even higher $\mathrm{x}$-ray attenuation coefficients than that of the bones. A practical example is shown in Fig. 4, where the stool in the colon has such a property.

Building intensity distribution of body wall voxels. Once the skin surface and the bones are identified, the voxels

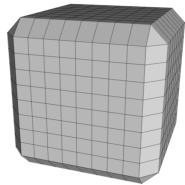

(a)

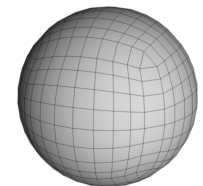

(b)
Fig. 2. Quadrilateral mesh on (a) a cube and (b) a sphere.

between the two can be extracted. To do this, for each point on the skin surface, a ray is projected along the inward surface normal direction until it meets a bone voxel. The voxels along the ray definitely belong to the body wall and are extracted (Fig. 1(a)). If a ray cannot find any bone voxel within certain distance, all the voxels along the ray are discarded. To reduce the number of extracted voxels, uniform sampling of the extracted voxels can be performed.

In general, most of the parametric or non-parametric distribution functions are suitable for building the intensity distribution model. In our work, a Gaussian mixture model (GMM) containing 3 Gaussians is used to serve this purpose, where each Gaussian approximates the intensity distribution of one type of tissue (i.e., skin, fat and muscle). The parameters of the GMM are estimated using the Expectation Maximization (EM) method. An example of the recovered GMM is shown in Fig. 1(b). The recovered GMM will be used for correspondence search in the deformation stage.

\subsection{Registration by 3D Flipping-free Deformation}

Based on the extracted features, this stage deforms a surface mesh, making it registered to the inner boundary of the abdominal wall. Essentially, the deformation of a mesh model $M$ can be defined non-parametrically as the displacement $\mathcal{D}$ : $\mathbf{u}_{i} \rightarrow \mathbf{v}_{i}$ where $\mathbf{u}_{i}$ is a mesh vertex and $\mathbf{v}_{i}$ is the target location of $\mathbf{u}_{i}$. However, naively deforming a surface mesh based on corresponding points is prone to the flipping problem as explained in [7]. In order to solve this problem, a special quadrilateral mesh is initially defined on a cube (Fig. 2(a)), then projected onto a sphere (Fig. 2(b)). Each vertex has exactly 4 neighboring vertices, and 3 orthogonal contour groups can also be defined on the mesh. These internal properties contribute to an easier solution of the flipping problem during deformation. This stage iteratively performs correspondence search for mesh vertices, flipping detection/resolution and deformation of the mesh until convergence.

Correspondence search. This step searches for each vertex $\mathbf{u}_{i}$ on the model $M$ a possible corresponding point $\mathbf{v}_{i}$ on target volume $T$ along the projection line $P\left(\mathbf{u}_{i}\right)$. The direction of $P\left(\mathbf{u}_{i}\right)$ can be defined as the surface normal at $\mathbf{u}_{i}$.

The ideal $\mathbf{v}_{i}$ is located on either the inner boundary of the muscle layer or the bones. Therefore, the algorithm can start from $\mathbf{u}_{i}$ (inside the abdominal wall), and estimate for $\mathbf{v}_{i}$ along a ray going outwards. $\mathbf{v}_{i}$ can be the first bone voxel 


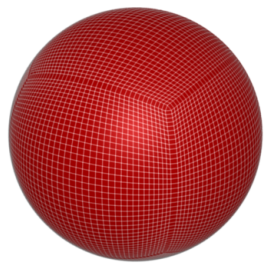

(a)

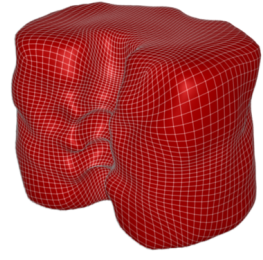

(c)

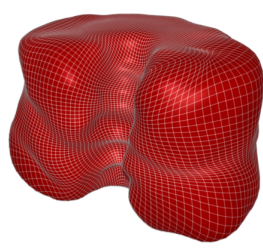

(e)

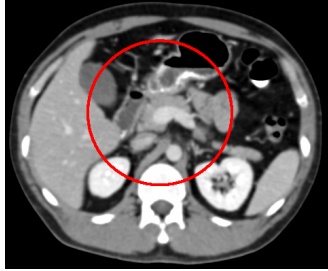

(b)

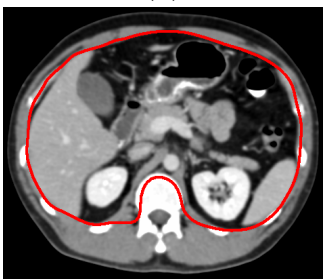

(d)

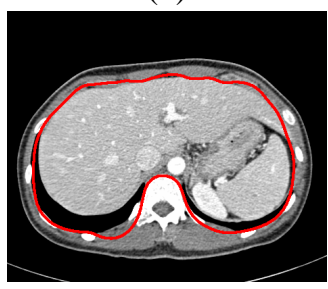

(f)
Fig. 3. Initial 3D quadrilateral mesh (a) and its $2 \mathrm{D}$ view (b) in one axial slice. Extracted 3D surfaces $(c, e)$ and their respective $2 \mathrm{D}$ axial views $(\mathrm{d}, \mathrm{f})$ for sample $\mathrm{CT}$ volume Images.

or muscle voxel. To ensure robust estimation of muscle, a voxel is treated as muscle only if its subsequent consecutive $n$ voxels are all within a standard deviation of the computed Gaussian distribution. Larger $n$ guarantees more robust estimation, since the joint probability of making false estimation goes down significantly with larger $n$. However $n$ cannot be too large because the muscle layer has limited thickness. Usually $n=5$ will suffice.

Flipping detection \& resolution. The flipping of a mesh cell after mesh deformation can be characterized by the flipping of at least one of its edges. The edge flipping can be detected by checking the following condition:

$$
\frac{\mathbf{u}_{i}-\mathbf{u}_{j}}{\left\|\mathbf{u}_{i}-\mathbf{u}_{j}\right\|} \cdot \frac{\mathbf{v}_{i}-\mathbf{v}_{j}}{\left\|\mathbf{v}_{i}-\mathbf{v}_{j}\right\|} \leq \tau
$$

where $\mathbf{u}_{i}$ and $\mathbf{u}_{j}$ denote two neighbors on a closed contour $C$ of mesh model $M, \mathbf{v}_{i}$ and $\mathbf{v}_{j}$ denote their respective corresponding points on the target $T$, and $\tau \in[0,1)$ is a predefined threshold. This condition essentially states that the orientation of $\mathbf{u}_{i} \mathbf{u}_{j}$ and $\mathbf{v}_{i} \mathbf{v}_{j}$ should not differ significantly. Note that each vertex $\mathbf{u}_{i}$ is an intersection of two closed contours on $M$. As a result, it will undergo two rounds of flip detection checks as the algorithm iterates.

Once flipping is detected along certain contour, correspondence for consecutive vertices causing flipping will be

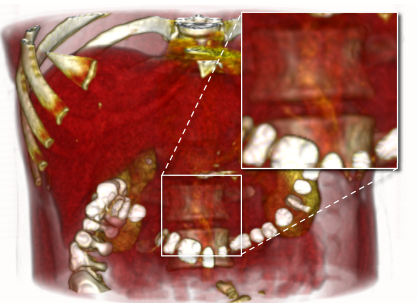

(a)

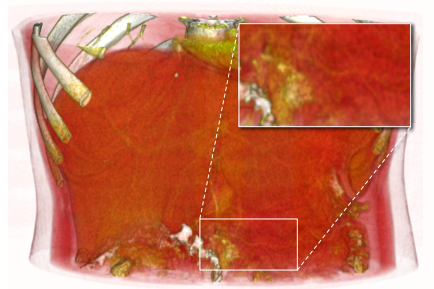

(c)

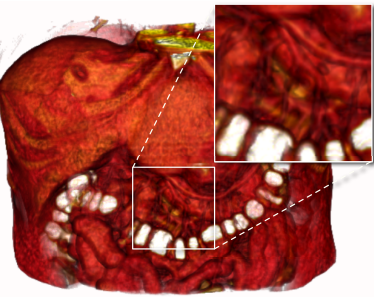

(b)

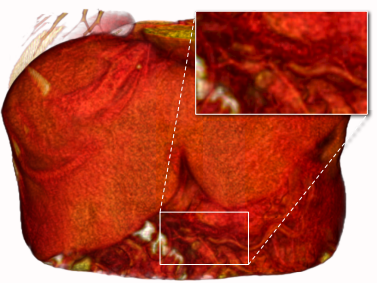

(d)
Fig. 4. Volume rendering of two sample CT volumes by setting the abdominal wall to transparent $(\mathrm{a}, \mathrm{c})$. Volume rendering of organs after removing the abdominal walls (c,d). Some of the organs such as colons and blood vessels that cannot be visualized in the former can be clearly visualized in the latter. Best viewed in color.

set to null except the middle one of them. The vertices without proper corresponding points initially may get their correspondence later on by means of interpolation.

This step creates valid correspondence without flipping for most of the vertices on the mesh. For vertices without valid correspondence, their displacements are solved by Laplacian deformation [8]. For details of the flipping-free 3D deformation, please refer to our previous work in [7].

Laplacian deformation. During deformation, nonflipping mesh vertices together with their corresponding points are regarded as positional constraints. The other mesh vertices are displaced according to the generic shape constraints incorporated into Laplacian deformation. These shape constraints include Laplacian preservation, surface smoothness, and uniform vertex distribution.

In deforming the mesh, instead of moving the mesh vertex from $\mathbf{u}_{i}$ to $\mathbf{v}_{i}$ in one time step, the algorithm can also move $\mathbf{u}_{i}$ by a fraction of the distance to $\mathbf{v}_{i}$, for instance

$$
\mathbf{u}_{i} \leftarrow(1-\lambda) \mathbf{u}_{i}+\lambda \mathbf{v}_{i}
$$

where $\lambda \in(0,1)$ is the step size. In general, choosing a smaller $\lambda$ may help keep the deformed mesh smoother, and to distribute vertices on the surface of the mesh better.

\section{EXPERIMENTS AND APPLICATIONS}

Our abdominal wall removal algorithm was tested on $10 \mathrm{ab}$ dominal CT volumes, each containing around 200 slices of 


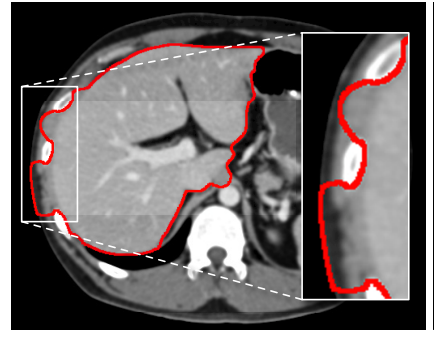

(a)

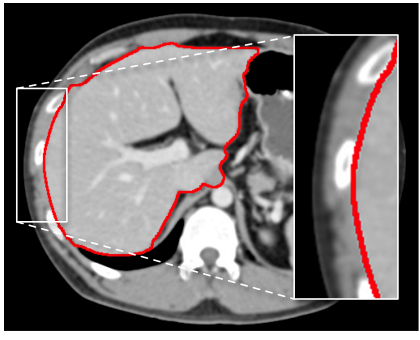

(b)
Fig. 5. Segmentation results of the snake algorithm with (a) and without (b) the abdominal wall.

DICOM images of thickness $1 \mathrm{~mm}$. The algorithm was initialized by placing a quadrilateral spherical mesh (Fig. 3(a,d)) with appropriate size inside the body. In the feature extraction stage, around 30k sample points were collected. Construction of their intensity GMM took about 5 seconds. The deformation of the quadrilateral mesh containing around 10k vertices took 15 iterations with a step size of $\lambda=0.4$ in $25 \mathrm{sec}-$ onds. The experiment was performed on an Intel Xeon $2 \mathrm{GHz}$ computer with $4 \mathrm{G}$ memory. The extracted inner boundary of the abdominal wall is represented as a surface mesh. Sample results of the extracted inner boundaries of the abdominal walls for two input CT volumes are shown in Fig. 3(b,e) and Fig 3(c,f) respectively, in both 3D view and 2D axial view.

Once the surface of the abdominal wall is extracted, the wall voxels can be easily removed since they are all located outside of the surface. Abdominal wall removal can be applied to visualization and segmentation of the organs.

\subsection{Visualization of Inner Organs}

Two examples for the volume rendering of CT images are shown in Fig. 4. In Fig. 4(a) and (c), the opacity transfer functions were adjusted so that the abdominal walls appear transparent and some of the organs can be observed. Since the opacity transfer functions were applied globally across the whole input volume, other inner organs which have similar voxel intensities such as colons and blood vessels also became transparent and cannot be observed. In contrast, after removing the abdominal walls by the proposed algorithm, they can be clearly visualized as shown in Fig. 4(b) and (d).

\subsection{Segmentation of Organs}

Removal of the abdominal wall reduces the search space for an algorithm segmenting organs, leading to higher efficiency and accuracy of the algorithm in general. It also removes undesired features which may mislead the segmentation algorithm. To demonstrate, we compared 2D liver segmentation results using the snake algorithm before and after removing the abdominal wall. The snake algorithm for both cases had exactly the same parameters and converged in 20 iterations.
The initial snake was initialized with the manually segmented liver contour. As shown in Fig. 5(a), before removing the abdominal wall, even though the algorithm was initialized with the ground truth, it was still attracted to the edges produced by the wall. In contrast, Fig. 5(b) shows a good segmentation result after removing the wall. Essentially, removal of the abdominal wall can help not only the snake algorithm, but also all the other algorithms to segment the inner organs. Similar facilitations were obtained for other segmentation algorithms such as the level set method.

\section{CONCLUSIONS}

This paper presented an abdominal wall removal algorithm to help to visualize and segment inner organs in the CT volume. The proposed algorithm is based on the estimation of intensity distribution of voxels inside the body wall, followed by the flipping-free 3D deformation of a quadrilateral spherical mesh. By removing the wall in the 3D volume, the organs in the abdomen can be exposed and visualized directly. It also makes segmentation more accurate and efficient by reducing the search space of the segmentation algorithm.

\section{REFERENCES}

[1] M. Kass, A. Witkin, and D. Terzopoulos, "Snakes: Active contour models," IJCV, vol. 1, no. 4, pp. 321-331, 1988.

[2] J. A. Sethian, Level Set Methods, Cambridge University Press, 1996.

[3] S. J. Osher and R. P. Fedkiw, Level Set Methods and Dynamic Implicit Surfaces, Springer, 2002.

[4] S. Maeda, M. Komatsu, H. Kim, A. Yamamoto, and K. Okuda, "Automatic segmentation of liver region employing rib cage and its 3-D displaying," in Proc. of SICE-ICASE, 2006, pp. 1465-1468.

[5] T. Klinder, C. Lorenz, J. von Berg, S. P. M. Dries, T. Bülow, and J. Ostermann, "Automated model-based rib cage segmentation and labeling in CT images," in MICCAI, 2007, vol. 4792, pp. 195-202.

[6] S. Suzuki and K. Abe, "Topological structural analysis of digital binary images by border following," CVGIP, vol. 30, pp. 32-46, 1985.

[7] F. Ding and W. K. Leow, "Robust and efficient 3D volume image segmentation by flipping-free mesh deformation and registration," Tech. Rep., National University of Singapore, 2008.

[8] O. Sorkine, Y. Lipman, D. Cohen-Or, M. Alexa, C. Rössl, and H. Seidel, "Laplacian surface editing," in Proc. of Eurographics. 2004, pp. 179-188, ACM Press. 